\title{
Quand le discours pédagogique entrave la construction des usages littéraciés du langage
}

\section{Élisabeth Bautier}

\section{(2) OpenEdition}

\section{Journals}

\section{Édition électronique}

URL : http://journals.openedition.org/pratiques/1378

DOI : $10.4000 /$ pratiques. 1378

ISSN : 2425-2042

\section{Éditeur}

Centre de recherche sur les médiations (CREM)

\section{Édition imprimée}

Date de publication : 15 décembre 2009

Pagination : 11-26

\section{Référence électronique}

Élisabeth Bautier, «Quand le discours pédagogique entrave la construction des usages littéraciés du langage », Pratiques [En ligne], 143-144 | 2009, mis en ligne le 19 juin 2014, consulté le 30 avril 2019. URL : http://journals.openedition.org/pratiques/1378; DOI : 10.4000/pratiques.1378

(c) Tous droits réservés 


\title{
Quand le discours pédagogique entrave la construction des usages littéraciés du langage
}

\author{
Élisabeth Bautier \\ Équipe Circeft-Escol, Réseau RESEIDA \\ Université Paris 8
}

La thématique de ce numéro conduit à développer ici deux questionnements étroitement liés : celui de l'acquisition de la littéracie et celui des savoirs curriculaires de l'école d'aujourd'hui ; ce double questionnement est en effet nécessaire lorsque, comme c'est le cas ici, l'appropriation, le codage des savoirs, leur traitement, leur écriture, sont travaillés dans la perspective du renforcement ou de la réduction des inégalités sociales d'apprentissages scolaires.

Lorsque l'on observe et analyse les situations de travail construites pour les élèves par les pratiques de classe en maternelle, puis en primaire, mais aussi lorsqu'on analyse des manuels scolaires, deux constats peuvent être faits de portée relativement générale ${ }^{(1)}$. D'une part, certains élèves, surtout lorsqu'ils sont issus de milieux populaires, sont mis en difficulté par les attendus scolaires ; ils ne participent pas des apprentissages prévus par l'enseignant et que d'autres élèves effectuent. D'autre part, il nous semble possible d'interpréter les situations étudiées comme relevant simultanément de l'évidence du partage par tous les élèves d'un traitement littéracié des situations de travail, des documents, des savoirs, et d'un cadrage des situations et d'un accompagnement linguistique et langagier qui va jusqu'à rendre la distinction écrit/oral opaque pour les élèves, brouillant le registre de travail attendu. Tout se passe comme si les exigences en matière de familiarité de plus en plus précoce avec une littéracie étendue et une raison graphique ne cessaient de s'accroître et que, dans le même temps, les conditions de cette socialisation cognitive étaient, paradoxalement, de moins en moins assurées dans les premiers moments de la scolarité. Ce paradoxe, et les apprentissages non effectués qui en sont la conséquence, pourraient expliquer la permanence, voire l'ac-

(1) L'expression « relativement générale » signifie que les situations auxquelles nous faisons référence sont très récurrentes actuellement, elles ont fait l'objet de nombreuses descriptions convergentes de différentes recherches depuis plusieurs années (il s'agit par exemple des travaux de l'équipe Escol-Circeft, ceux du réseau RESEIDA, de la thèse de Caffieaux, de l'ouvrage de Amigues et Zerbato-Poudou...), suffisamment convergentes pour que les descriptions qui suivent aient une valeur qui dépasse le contexte des exemples retenus ici. Il ne s'agit pas pour autant d'affirmer qu'il n'y a pas des variations selon les enseignants, les situations de classe, la population d'élèves... 
croissement, de la difficulté scolaire chez une population d'élèves, toujours la même en termes de situation sociale, malgré, ou plutôt du fait, des évolutions didactiques et curriculaires.

Afin d'étayer ces hypothèses, nous développerons dans un premier temps les conceptions de la littéracie, qui sous-tendent le propos, dans un second temps, ce qui nous semble être identifiable à des modifications curriculaires importantes. Dans la mesure où les contenus d'apprentissage sont indissociables de leur mise en œuvre dans des formes pédagogiques et celles-ci ayant également évolué pour faire une large place aux échanges langagiers dans la classe, nous illustrerons ensuite nos interprétations par des analyses du discours pédagogique.

\section{Raison graphique et littéracie étendue}

Il peut apparaître surprenant quand il s'agit de savoirs de l'écrit, élaborés dans l'écrit et de leurs codages par l'écriture, de travailler aux niveaux scolaires les plus élémentaires que sont la maternelle et l'école primaire. Notre choix s'explique par la fonction de socialisation cognitive de l'école ${ }^{(2)}$. L'école, par ses formes de travail, les objets auxquels elle confronte les élèves, la hiérarchie des savoirs et des activités qu'elle leur donne à voir, construit des modes de faire, de dire, de penser spécifiques. Les premiers apprentissages constituent pour l'élève une socialisation cognitive l'introduisant (ou non) à la littéracie, c'est-à-dire à une forme simultanée de pensée, de langue et de langage nécessaire aux apprentissages scolaires et au-delà à l'intégration sociale. La question que nous soulevons est celle des élèves qui passent à côté de cette socialisation cognitive et donc de la visibilité des attendus scolaires et des acquisitions possibles.

Même si nous ne développons pas ici les difficultés d'acquisition du code écrit, des équivalences graphie-phonie, de l'identification de la composition de la chaîne parlée, d'autres l'ont fait ${ }^{(3)}$, loin de les minimiser dans ces premiers moments de la scolarité, nous souhaitons au contraire, non seulement insister sur le fait que cet aspect de l'apprentissage de l'écrit est le plus souvent pensé comme neutre, comme « purement » technique, « purement» cognitif, quand, comme tous les savoirs, il s'agit d'une construction sociale, qui suppose et implique donc des conditions spécifiques à son acquisition. En revanche, nous pouvons souligner combien l'utilisation ordinaire de la raison graphique dès la maternelle par le biais de supports présentant des tableaux, des listes et une occupation de l'espace graphique à visée cognitive relevant de cette raison ignore que celle-ci n'a rien de spontané et participe d'une différenciation sociale à l'œuvre dès les premiers moments de la scolarité ${ }^{(4)}$.

La forme littéraciée d'usages du langage va, en se développant, permettre à l'enfant, à l'élève, de participer des modes actuellement dominants de traitement des savoirs scolaires et non scolaires, des relations sociales, des modes de construction des raisonnements qui portent sur la compréhension de phénomènes, de processus, plus que sur la mémorisation des faits ou des définitions. Il faut encore inclure dans ce que construit la familiarité avec la culture écrite scolarisée, l’habi-

(2) Forquin (2008)

(3) Pour une synthèse théorique, on peut se référer à la thèse de Caffieux, L'entrée dans l'écrit : l'influence des pratiques d'enseignement à l'école maternelle, 2007, ULB, Bruxelles, sous la direction de Rey.

(4) Voir Joigneaux, Des processus de différenciation dès l'école maternelle. Historicités plurielles et inégalité scolaire. Thèse de doctorat, juillet 2009, université Paris 8 ; Gachet-Delaborde, Formes et sens de l'univers graphique en maternelle. Thèse de doctorat, novembre 2009, Université de Metz. 
tude des mobilisations et des mises en relation de domaines et références hétérogènes, de leur rapprochement sous leur diversité apparente, parce que le niveau de connaissance visé n'est pas le niveau conjoncturel, celui de la réalisation de l'activité telle qu'elle est présentée dans le moment de son effectuation, mais celui du savoir générique.

La littéracie telle qu'elle est entendue ici est donc non seulement une acquisition des formes écrites, mais aussi un rapport à la langue, à son écriture, le produit des transformations sociales et cognitives que l'usage de l'écrit a produits dans la société et qui ont conduit à étendre le rapport symbolique et médiatisé à la langue aux objets du monde et à l'expérience elle-même, y compris dans les situations d'échange oral. Par littératie étendue (full literacy), nous désignons donc les usages oraux et écrits du langage développés par une fréquentation familière et fréquente de l'écrit et de l'écriture dans une acception qui ne les réduit ni à la maîtrise des outils techniques de l'écrit (graphie, morphologie et syntaxe spécifiques), ni aux usages de l'écrit construits historiquement dans une société donnée, comme le stockage de l'information, les notations et enregistrements divers de données, qui remplacent la mémoire et qui se sont inscrits peu à peu dans les usages sociaux de cette société (Reuter, 2006). La littératie étendue désigne les possibilités intellectuelles développées par l'usage heuristique de l'écrit, de l'écrit d'élaboration, celui qui suppose aussi l'interprétation du lecteur, qui n'est ni dans une transparence par rapport au réel, ni une consignation de l'oral. La littératie étendue correspond à ces usages de l'écrit qui, au cours du temps, ont contribué aux constructions et activités intellectuelles qui élaborent des lois générales et non des seules narrations d'expériences locales et spécifiques, qui transforment les expériences en savoirs, qui permettent de tisser ensemble (de penser et d'écrire avec) différentes voix d'origine diverses, savantes et vulgaires, orales et écrites. C'est bien de ces usages de ressaisie, de reprise dont il s'agit quand les élèves sont censés écrire un texte qui s'inscrit dans une discipline donnée en mobilisant des sources aussi diverses et hétérogènes que la parole enseignante, des connaissances tirées des différents médias, les échanges au sein de la classe, l'observation de tel ou tel document et son commentaire explicite ou non... L'écrit long de savoir n'est donc pas « seulement » notation écrite, restitution, mise en œuvre de techniques d'écriture, mais élaboration et acquisition de modalités de pensée spécifiques : intériorisation, distance, travail du document. Il permet la secondarisation des objets du monde (Bautier, 2006), la prise de conscience de la tâche à effectuer dont on est l'auteur, conscience qu'il s'agit de penser-écrire quelque chose et non de répondre à une question. Il permet encore la pratique du commentaire. Cette énumération de la puissance cognitive et langagière de l'écrit explique la prégnance de la littéracie étendue dans les attentes et habitudes scolaires, elle leur sont en effet sous-jacentes et vraisemblablement sans une claire conscience des incidences de la part des enseignants. Ainsi, l'usage implicite du genre second du langage (Bakhtine, 1984) est largement dominant dès l'école maternelle (l'autonymie peut en être un exemple) et sans que celui-ci fasse l'objet d'une attention explicite comme objet d'apprentissage pour les élèves de la part de l'enseignant.

Si nous insistons sur les conséquences de l'écrit dans les habitudes cognitives, cela ne doit pas laisser dans l'ombre les conséquences sociales de l'influence de la littéracie étendue sur les pratiques ordinaires et les valeurs qui les sous-tendent. Ainsi, la référence au texte se fait au détriment de la parole et de l'expérience, le travail interprétatif du langage au détriment d'une conception du langage qui dit le « vrai » du monde, d'où le caractère paradoxal des situations de débat et d'échanges, de certains dialogues scolaires, dont nous donnons des exemples ci-après.

Sans doute, peut-on penser qu'il n'y a là rien de nouveau si l'on se réfère aux 
pratiques construites dans la tradition des humanités classiques. Mais tel n'est pas le cas si on prend en considération le public concerné aujourd'hui par les études longues. Les élèves des lycées, jusque dans les années quatre-vingt, étaient issus de milieux sociaux favorisés et eux-mêmes lettrés, tel n'est pas le cas aujourd'hui. De plus, dans la tradition des humanités, la référence constante aux textes, les différents exercices d'entraînement à l'écrit, l'écrit long en particulier, de répétition, d'imitation, l'accompagnement par l'enseignant dans le travail de commentaire constituaient autant de médiations pour une entrée progressive dans la fréquentation et l'appropriation des spécificités du rapport scolaire à l'écrit. Le paradoxe de la société littéraciée contemporaine, c'est qu'elle suppose partagées des habitudes construites dans la fréquentation de l'écrit même quand celles-ci n'ont pu l'être encore ou ne peuvent l'être compte tenu des habitudes et histoire sociales des familles dont sont issus les élèves.

Ce qui nous intéresse ici, ce sont donc les conditions de l'apprentissage ou de l'appropriation scolaire de l'ensemble des dispositions cognitives et sociales que suppose cette littéracie et qui sont souvent invisibilisées, pour les enseignants comme pour les élèves, par les pratiques actuelles, tant dans la nécessité de leur apprentissage, que dans leur nécessaire mobilisation dans les activités proposées. Cette invisibilisation des objectifs de savoirs, des savoirs eux-mêmes tels que «pris » dans les activités scolaires et le discours qui les accompagne rend fréquent le leurre d'acquis que chacun pense avoir effectués et qui ne le sont pas ou sont des manières de faire avec les tâches demandées plus que des acquisitions transformatrices ; ce phénomène creuse les inégalités quand l'école maternelle et primaire pourrait (devrait) les réduire.

Lahire (2008) a largement étudié les difficultés scolaires des élèves de milieux populaires liées à leur rapport non littéracié à la langue quand les tâches, les exercices, 1'acquisition de la grammaire scolaire comme des formes narratives, tout ce qui fait productions scolaires suppose un rapport de familiarité avec cette culture écrite et le statut de langue-objet et non langue de communication qu'elle construit. Lahire souligne ainsi les malentendus ${ }^{(5)}$ dont les élèves sont victimes et qui reposent sur la non-prise en compte par l'école du caractère sociologiquement construit de ce rapport au langage qu'elle sollicite.

Par rapport à ces travaux sur les difficultés scolaires des élèves de milieux populaires liées au caractère spécifiquement scriptural de l'univers scolaire, et que nous ne remettons nullement en question, nous développons une hypothèse qui permet de prendre en considération l'ensemble des situations de travail scolaire, au-delà des seuls moments consacrés à la production linguistique et langagière dans le champ disciplinaire de la langue maternelle. L'hypothèse repose sur les effets du cumul de deux phénomènes : cette évidence scolaire d'une culture écrite partagée par tous dès le plus jeune âge. Cette évidence se combine avec une familiarité, partagée au sein même de l'école, avec une langue qui n'est plus tout à fait le français standard de l'école et des apprentissages, langue grammatisée et justement fondée dans l'écrit, mais une langue de communication quotidienne, langue circulante, vernaculaire, qui véhicule le partage des références et des expériences. L'illusion de la connivence linguistique et de la familiarité de tous avec la langue écrite vient sans doute de la réduction de l'écart entre cet oral ordinaire et la langue standard du fait de la scolarisation massive et des médias. Comme nous espérons le montrer, le paradoxe ainsi construit, et que nous exprimons en termes bernsteiniens, se situe dans la cons-

(5) Au sens développé par Bautier et Rayou, 2009, Les inégalités d'apprentissage. Programmes, pratiques et malentendus scolaires. 
truction au quotidien de la classe d'un discours horizontal et de situations « particularistes », concrètes, à traiter " matériellement » dans un oral de l'oralité ordinaire, quand l'objectif réel est celui de la construction de significations universalistes, générales qui ne peuvent se construire que dans 1'élaboration que permet l'usage littéracié de la langue, y compris dans des échanges oraux.

\section{Littéracie étendue et changements curriculaires}

Pour comprendre les effets potentiellement négatifs pour une partie de la population scolaire de cette situation scolaire très contemporaine, elle doit être mise en relation avec les évolutions curriculaires qui, justement, touchent simultanément savoirs et langage. Nous avons déjà ailleurs (Bautier/Rayou, 2009) développé les relations étroites entre l'importance accrue des usages du langage dans la classe et l'affaiblissement des savoirs disciplinaires (code sériel chez Bernstein) au profit non seulement des savoirs de communication orale et écrite, mais aussi de savoirs transversaux et de nouveaux savoirs régionalisés ${ }^{(6)}$ (Bernstein, 2007) et surtout de la segmentation des savoirs liés à leur contextualisation de plus en plus grande et à leur transformation en compétences. Ces savoirs ne relèvent pas d'une inscription disciplinaire particulière, mais ils les supposent souvent ; ils supposent également la secondarisation de l'expérience et de la culture quotidienne médiatisée, ou plus largement de l'expérience culturelle et sociale des milieux fortement scolarisés, et la mise en relation de compétences et connaissances hétérogènes (il en est ainsi de l'introduction du cinéma à l'école, plus largement de l'art, de l'éducation à l'environnement ou à la santé). Le rôle du langage littéracié est central dans le processus de construction de ces savoirs, c'est lui qui est à l'œuvre dans sa fonction de mise en relation, de construction de la généricité et d'élaboration du « nouveau » par les élèves eux-mêmes.

Les modifications curriculaires (et pédagogiques) correspondent à des transformations sociales (Forquin, 2008), elles relèvent largement comme le souligne Young (2001) des nécessités sociales et économiques contemporaines, des nouvelles répartitions des tâches et des nouveaux métiers où il s'agit de façon récurrente d'établir et de maintenir des connexions, des interactions, des collaborations entre différents domaines de savoirs, de construire des liens, des échos. Ces « nécessités » constituent aujourd'hui une partie importante du curriculum scolaire et des savoirs à acquérir ; elles figurent d'ailleurs de façon récurrente dans le socle commun. C'est ainsi la mise en mots littéraciée du monde par la construction de relations entre domaines, objets scolaires et non scolaires, disciplinaires et régionalisés qui lui donne aujourd'hui de l'intelligibilité, bien au-delà des savoirs disciplinaires historiquement convoqués pour le comprendre. Il ne s'agit pas ici de mettre en question, a fortiori en cause, une adaptation curriculaire sans doute nécessaire. Il est vraisemblable que les savoirs « utiles » pour faire du lien social, utiles dans le processus d'intégration sociale, sont aujourd'hui davantage langagiers et cognitifs que patrimoniaux (Young, 2008).

On ne pourrait donc voir ici qu'une évolution curriculaire «normale » en ce que les savoirs sont le produit des évolutions sociales, techniques et assurent la compréhension du monde (Forquin, 2008). Cependant, et paradoxalement, si on se souvient des attaques dont les savoirs académiques, culturellement et socialement

(6) «Les régions sont construites par recontextualisation de catégories singulières (nous dirons ici des disciplines, NDLR) en ensembles plus larges qui opèrent à la fois dans le champ intellectuel et dans le champ de la pratique externe. [...] La régionalisation en tant que procédure discursive menace les cultures pédagogiques dominées par les catégories singulières et pose la question de la légitimité de ces cultures. », p. 93. 
construits, ont fait l'objet dans les travaux sur la reproduction sociale par l'école où la discrimination dont les milieux sociaux populaires étaient, de leur fait, l'objet, la prépondérance actuelle des savoirs situés dans les maniements langagiers littéraciés est plus discriminante encore, puisqu'elle invisibilise ce qui est savoir aujourd'hui. Elle situe le plus souvent dans les caractéristiques des sujets leur incompétence dans ces maniements, d'ailleurs souvent réduits à des savoir-faire techniques, ce qu'ils ne sont pas seulement ou à des évidences partagées dès que l'enfant est entré dans le langage. Cette invisibilisation accroît la domination éprouvée par certains élèves qui n'identifient pas l'objet des apprentissages. Ce curriculum actuel et sa mise en scène dans l'ordinaire des classes est particulièrement discriminant dans la mesure où ils conduisent à la sollicitation constante et à l'interpénétration des niveaux cognitif (au sein d'opérations et de travail cognitif), langagier, culturel, des savoirs et savoir-faire scolaires ; les différences entre élèves s'opèrent à chacun d'entre eux et les rendent justement peu identifiables du fait de cette interpénétration même. Les contenus de savoir sont aujourd'hui devenus flous ou du moins, sont des enchevêtrements de contenus disciplinaires, ponctuels le plus souvent, d'usages du langage, de raisonnements et de modes de faire procéduraux dans les activités.

Le curriculum latent est alors de plus en plus prégnant, en particulier, du fait des conceptions pédagogiques, mais le contenu de ce curriculum, « cet ensemble de compétences et de dispositions que l'on acquiert à l'école par expérience, imprégnation, familiarisation ou inculcation diffuse plutôt que par le biais de procédures pédagogiques explicites ou intentionnelles » (Forquin, 2008) n'est plus limité aux habitudes de travail, à la scolarisation au sens de "métier d'élève ", mais il correspond aux caractéristiques de ces contenus faiblement verbalisables, instituationnalisables et qui ne peuvent constituer un texte de savoir (Bernstein, 2007). Le développement des pratiques langagières et cognitives littéraciées relève en effet davantage de l'intériorisation que de l'explicitation.

Ce sont, en conséquence, les composantes des situations qui deviennent essentielles dans cette socialisation cognitive et langagière, ce qui conduit à les analyser dans leurs traits récurrents actuels, notre visée étant ici de pouvoir lier les dimensions macrosociologiques du curriculum que nous venons rapidement d'évoquer et les dimensions microsociologiques de sa mise en œuvre, dont les enseignants ne sont pas toujours conscients. Nous revenons ci-après sur des exemples de situations de classe dans le primaire ${ }^{(7)}$, mais nous pouvons dès à présent soulever la question déjà posée par Young (2008) de façon plus générale, concernant les relations entre les exercices pratiques, les manipulations, les activités ludiques si nombreux en maternelle et encore en primaire (où faire et apprendre sont souvent mis en équivalence) et les savoirs conceptuels. On peut certes avec Vygotski situer dans l'activité pratique la genèse de la pensée conceptuelle, mais les processus par lesquels ces activités sont transformées dans et par la théorie ou tout au moins conscientisées échappent à la description. Sans doute sont-ils en partie liés à l'indépendance du champ dont font preuve (ou non) les sujets, processus dont les travaux en psychologie différentielle ont montré le caractère justement différenciateur.

(7) Le corpus auquel nous nous référons a été constitué au cours de plusieurs années de recherche dans les classes de Grande section, de Cours Préparatoire et de Cours Moyen par des membres du réseau RESEIDA, ces classes sont situées dans plusieurs académies et les enregistrements et transcriptions effectués correspondent à des observations dans la durée (plusieurs observations par semaine sur plusieurs semaines dans l'année) pour suivre des élèves sur deux ans le plus souvent. 


\section{Quand les situations de travail et le discours pédagogique rendent improbable la construction des savoirs littéraciés par tous les élèves}

Les développements précédents conduisent à analyser les pratiques et productions langagières scolaires de plusieurs points de vue étroitement liés.

Le point de vue des conditions d'une socialisation cognitive et langagière littéraciée, en d'autres termes, comment les pratiques et discours enseignants permettent d'introduire et d'apprendre aux élèves ces usages spécifiques du langage qui les conduisent à entrer dans les savoirs scolaires, à les penser et les nommer, à penser et nommer les activités cognitives qui les construisent.

Le point de vue des évolutions curriculaires, telles qu'elles apparaissent dans les mises en scènes des savoirs, les dispositifs, les évaluations, les discours qui les mettent en œuvre.

Nous ne développerons pas ici l'analyse des dispositifs pédagogiques qui, dans l'école primaire au moins, dominent les situations d'étude, ils ont été largement exposés ailleurs depuis quelques années ${ }^{(8)}$. Il nous suffit ici de rappeler 1'influence doxique des conceptions de l'apprentissage qui valorisent l'apprentissage au gré des activités et identifient le savoir à ce qui peut se construire dans l'expérience de cette activité. Mais la mise en œuvre au quotidien de ces conceptions confère à ces savoirs un caractère segmenté et pris dans leur contexte ${ }^{(9)}$ et dont l'appropriation est rarement le fait de tous les élèves car ils sont produits au fil de 1'activité et des échanges ; or ceux-ci se révèlent (voir ci-après) très rarement suffisamment contraignants pour orienter l'activité de tous dans la même direction.

Nous voudrions plus précisément tenter de caractériser le discours scolaire dominant actuellement dans ses effets potentiellement différenciateurs sur la socialisation cognitive et langagière littéraciée des élèves. Il n'est guère possible de dissocier les manières de faire et de dire des enseignants des contraintes multiples, voire contradictoires, qui pèsent sur l'ordinaire des classes concernant les objectifs de l'école elle-même. L'école maternelle est ainsi selon les textes officiels simultanément la première école où il est nécessaire que les élèves entrent dans les apprentissages scolaires, entrent dans l'écrit, mais où il est tout autant nécessaire qu'ils communiquent, s'expriment et entrent dans les modalités des échanges scolaires. Nous retrouvons les traces de ces objectifs souvent contradictoires dans la plupart des enregistrements et observations effectués dans les différentes classes de Grande Section et de Cours Préparatoire, mais encore de Cours Moyen dans lesquelles nous avons travaillé.

Si on tente de caractériser les différentes situations de travail rencontrées le plus fréquemment dans les classes quels que soient les objets de travail (lecture, découverte d'un texte, géographie, sciences, mathématiques, production écrite...), deux modalités pédagogiques sont identifiables.

- Le cours dialogué dont l'avancement est fondé sur un jeu constant et très rapide de question-réponse ; nous reprenons l'expression « cours dialogué » car elle fait partie des catégories descriptives des modes pédagogiques, mais aux niveaux scolaires étudiés, elle est abusive dans la mesure où justement, il n'y a pas toujours de « cours ", au sens de nouveaux savoirs à acquérir, où la visée cognitive du cours, son objet même et son maintien dans la durée ne sont pas toujours maintenus du fait des échanges eux-mêmes et de leurs caractéristiques.

- Les séances où les élèves ont une tâche à réaliser à partir d'un support, d'une

(8) Voir Bautier, dir., 2006.

(9) Voir le développement des effets des savoirs segmentés dans Karl Maton (2008). 
manipulation, d'une observation, celles-ci étant censées leur permettre de construire un savoir.

Paradoxalement peut-être, ces deux modalités ne sont pas à l'origine de comportements langagiers et linguistiques très différents de la part des enseignants du point de vue de la formation langagière, linguistique et cognitive des élèves.

Compte tenu de notre objet, nous avons centré nos analyses sur des éléments spécifiques susceptibles de permettre la construction des usages littéraciés. Il s'agit des éléments linguistiques de décontextualisation, de généralisation, des indices d'une langue d'apprentissage des productions « écrites » de savoir ; il s'agit également de l'usage d'une langue standard grammatisée, l'usage des mots des savoirs disciplinaires, des concepts, l'usage d'une métalangue et d'un métalangage et des formes de définition, d'explication, d'argumentation qu'ils permettent de déployer, de la nomination des activités cognitives nécessaires au travail à effectuer.

Par ailleurs et conformément au cadre théorique bernsteinien qui est le nôtre, nous avons voulu par le biais des notions de cadrage, de discours régulateur vs instructeur, identifier la caractère visible ou invisible des objectifs cognitifs des moments et des séances étudiés, les façons de tenir un objet constant ou non. Enfin, la distinction de Bernstein lors de ses analyses du discours pédagogique entre discours horizontal et discours vertical permet également de construire une catégorisation plus complète du discours enseignant. Le discours horizontal est le genre largement dominant; tel que le définit Bernstein (2007), c'est un discours local, enraciné dans les procédures pratiques, totalement contextualisé, un oral qui traite les savoirs de façon segmentée, c'est-à-dire en situation, liés à une tâche particulière : "L'organisation des savoirs dans DH conduit à des acquisitions segmentées...(cette) pédagogie s'exerce généralement dans des relations de face à face, fortement chargées affectivement [...] cette pédagogie porte davantage sur une compétence ordinaire plutôt que sur une "acquisition" classificatrice. " (10)

Notre objectif dans les exemples qui suivent n'est pas de décrire une classe particulière, un mode de faire spécifique d'un type d'enseignant, mais d'illustrer des phénomènes récurrents, le plus souvent cumulés dans la pratique de l'enseignant, et qui par leur cumul gênent justement les apprentissages des élèves les moins familiers socialement des usages littéraciés. C'est donc la récurrence des éléments caractéristiques ci-après qui nous permet d'identifier un genre discursif scolaire actuel dominant en maternelle et dans le primaire.

\subsection{Des échanges plus communicationnels que cognitifs}

Nous avons déjà évoqué l'affaiblissement des modalités transmissives des savoirs, et même leur rejet à l'école maternelle et primaire, au profit de leur élaboration par les élèves à partir des situations et des objets auxquels 1'enseignant les confronte. Ces mises en situation constituent une double contextualisation qui ancre ce qui est « appris » dans le moment de son évocation (et non dans une progression où le lien avec d'autres éléments de savoir participe de ce qui le constitue) ou dans la tâche en train d'être effectuée ; un autre type de contextualisation peut éga-

(10) On peut rapprocher la description du DH de celle du discours quotidien ou d'opinion qu'Astolfi, Peterfalvi et Vérin (1998) opposent au discours scientifique, fondé dans l'écrit : l'interlocuteur est un individu ou un groupe identifiable auquel on s'adresse, la validité du discours est locale et actuelle, on parle, on dialogue entre interlocuteurs, la subjectivité peut se déployer à la recherche d'une intersubjectivité, la situation est égalitaire, la logique le plus souvent naturelle, rien n'est précis ni préalablement défini dans le discours, les circonstances sont déterminantes pour la compréhension. 
lement être sollicité par l'enseignant quand il fait appel à la connaissance ou l'expérience des élèves (voir ci-après). De telles démarches laissent le plus souvent implicite et à la charge des élèves l'activité de décontextualisation, mais elles entraînent également, ou vont au moins de pair avec, des caractéristiques langagières et linguistiques qui les en éloignent.

Très rares en effet sont les consignes de travail et les cadrages de la part de l'enseignant qui situent le travail dans une perspective qui dépasse la situation immédiate et l'inscrit soit dans une continuité d'apprentissage, dans un approfondissement ou une révision. Les échanges qui constituent souvent l'essentiel d'une séance sont produits dans une langue quotidienne, dans un mode conversationnel de la part des enseignants comme de la part des élèves. Ils ne présentent en effet de la part de ce dernier ni explication précise, ni définition, ni argumentation qui situerait le propos dans un apprentissage classificatoire ; ils se déroulent le plus souvent au fil des interventions de chaque élève et de leurs interactions, dans un discours horizontal, même si c'est l'enseignant qui conduit le dialogue. Un tel enchaînement ne permet pas d'inscrire la séance dans une cohérence notionnelle ou conceptuelle. De plus, la langue ordinaire qui prévaut, celle du vernaculaire de l'oralité quotidienne, ne permet guère aux élèves d'interpréter la situation comme étant celle d'un travail d'élaboration, d'un usage langagier spécifique à celui-ci. Les verbes désignant consignes ou activités cognitives sont ainsi des verbes de la vie « ordinaire », les contraintes de travail et les évaluations ou validations apparaissant sur un registre de dialogue entre pairs, sur le mode de l'accord ou du désaccord, sans référence à des critères de pertinence disciplinaire ou de raisonnement; elles sont davantage fondées sur des critères d'effectuation normées des tâches (coller une étiquette-mot au bon endroit, par exemple), sans que les raisons cognitives en soient verbalisées ou enseignées :

\author{
Ens : Vous allez voir comment on calcule les pourcentages \\ Ens : On est d'accord \\ Ens: Ca vous va? \\ Ens : Dans l'ensemble c'est bon \\ Ens: On vous demande de colorier les flèches qui vous semblent bonnes
}

Les formulations erronées sur le plan disciplinaire des élèves peuvent être reprises en l'état par l'enseignant :

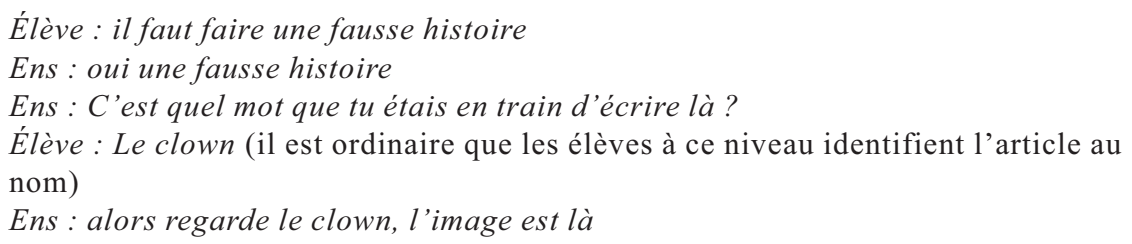

ou corrigées de façon non explicite et entretenant souvent la confusion entre le mot et son référent :
Ens: ici, c'est quel animal? (il y a une image)
Élève : Le lion
Ens : Lion, où est-ce que tu vas écrire le mot lion?

On peut noter ici l'exemple d'une aide qui, avec cet élève en difficulté, devient une aide à coller au bon endroit et non à comprendre ce qui a trait au code écrit et à l'identification des lettres.

Les réponses données à des questions de certains élèves concernant du vocabulaire, peuvent ainsi également relever de la conversation ordinaire, y compris dans 
les formes syntaxiques de l'oralité, non d'une forme définitoire ou explicatives. Ces dernières sont ainsi très peu données à entendre et à apprendre aux élèves et ne constituent pas les ressources d'un vocabulaire de langue « écrite » (au sens défini précédemment) :

Elève: qu'est-ce que la cohue?

Ens : la cohue, c'est-à-dire le bazar, que on entende plus, ça se bouscule, que ce soit le bazar

Les concepts ou les mots des savoirs sont dans le même sens évités ou paraphrasés dans cette langue orale ordinaire :

Ens : un losange, c'est comme si on avait tiré sur un carré

Ens : les diagonales se croisent en plein milieu

Ens : la proportionnalité, les pourcentages, c'est pareil

Ens : des forces de sens opposées, ça veut dire un petit peu de tous les côtés, ça se retire

Ens : le trapèze, il y a 2 côtés parallèles, c'est tout

(Exemples de classes de CM.)

Ens : c'est une fille (pour signifier qu'il s'agit de mettre un adjectif au féminin)

(Exemple de classe de GS.)

L'exploration de nouveaux domaines de savoirs se faisant dans cette langue, pour des raisons d'aide et de facilitation du dialogue exploratoire, certains élèves sont conduits à mobiliser l'univers de l'expérience également quotidienne, ordinaire, et non celui des savoirs en train de se construire.

Un exemple caricatural est donné par cet élève à qui l'on demande comment mangeaient les hommes préhistoriques, et qui répond qu'ils allaient à Carrefour. La construction dans la classe par un langage littéracié d'un univers de savoir décontextualisé de l'expérience des élèves éviterait plus aisément ce brouillage des références qui en pénalise certains gravement.

On pourrait certes attribuer ces énoncés à des enseignants ne maîtrisant pas le domaine de savoir et restant volontairement dans l'approximation, donc un phénomène marginal, mais les exemples de ce type sont trop nombreux, à différents niveaux scolaires, chez des enseignants très différents, enseignant dans des académies différentes, pour ne pas envisager une cause plus générale. En cohérence avec l'ensemble des indices présentés, il s'agit bien davantage d'une modification du rapport à la langue scolaire comme langue des savoirs. Comme s'il s'agissait de rejeter une situation (une langue) qui, lorsqu'elle s'imposait aux élèves, pouvait être vécue comme un obstacle, un indice de leur éloignement de la culture et des attentes scolaires. Mais à l'inverse actuellement, le discours pédagogique comporte trop peu de formes linguistiques et discursives métalangagières, définitoires, à visée cognitive, trop peu de validation argumentée... pour introduire les élèves à une langue, à des usages qui vont leur permettre d'avoir les ressources pour produire les activités qui leur sont aujourd'hui demandées, ou pour reformuler ultérieurement et par écrit dans un texte de savoir ce qui a pu être appris. Sont ainsi très rares les termes qui désignent les opérations cognitives à mettre en œuvre et qui constituent néanmoins l'apprentissage réel qui sous-tend, consciemment ou non l'activité.

En Grande Section de maternelle, dans une activité où il s'agit d'apprendre à l'élève à reproduire le même collier (nombre de perles et couleur) et à le vérifier, l'enseignant n'utilisera jamais le verbe "comparer" mais toujours "faire pareil », "voir si c'est pareil ». Au motif d'une compréhension plus aisée pour les élèves. L'expression « faire pareil » est ainsi le générique d'activités au demeurant 
plurielles du point de vue de ce qu'elles sont censées mobiliser (identification d'indices fins, de mots, de nombre d'objets, de couleur...) ; la distinction des activités précises n'est pas verbalisée.



La langue de l'école est identifiée à la langue vernaculaire pour échanger et communiquer. Tout semble alors se passer, au moins pour les élèves en difficulté, comme si les échanges étaient en eux-mêmes leur propre finalité : celle qui installe un climat de connivence, de confiance, sans qu'un apprentissage vienne les justifier, s'il les sous-tend, il ne les cadre pas. Et la rapidité des échanges qui construisent parfois des savoirs ou connaissances nouvelles en accompagnement d'une manipulation ou effectuation d'une tâche ne permet pas aux élèves en difficulté de passer, comme l'enseignant le souhaite ou le prévoit, de la manipulation, à la verbalisation, puis à l'écriture.

Cette interprétation en faveur de la prégnance d'un discours horizontal peut expliquer les phrases inachevées, les erreurs des élèves non relevées par l'enseignant, l'absence d'un temps de pause pour l'explication d'un mot, d'une connaissance ou d'un savoir non partagés, l'absence générale de reprise des énoncés des élèves, de demande d'explication, de justification de la part de l'enseignant, qui pourtant seraient nécessaires si un apprentissage était l'objectif de ces échanges. Une telle interprétation est peut-être plus difficile pour expliquer l'occurrence unique d'un énoncé, fût-il celui d'une règle, d'une procédure, d'un savoir :

Ens en CP: « $i$ » et « $n »$, ça fait « in», dans « homme» le «h» est une lettre muette, c'est « $t$ » à la fin avec « il », c'est « $t »$

Un énoncé unique ne suffit certainement pas à son acquisition, surtout quand les remarques sont opportunistes, puisqu'elles dépendent soit des productions des élèves, soit des éléments apparaissant dans le document lu. Loin de réitérations, de révisions ou répétitions, les énoncés du dialogue pédagogique se succèdent très rapidement, comme si formes et contenus étaient partagés par tous et qu'il s'agissait d'un simple rappel d'évidences. Ce qui n'est évidemment pas le cas. Ou comme si l'enseignement, le discours au nom d'un savoir, était considéré en contradiction avec le mode d'enseignement actuellement valorisé.

Ce climat de dialogue entre pairs au cours duquel l'enseignant se situe souvent comme membre du groupe au même titre que les élèves, abandonnant ainsi explicitement une position de savoir et les formes discursives d'échanges conversationnels horizontaux auxquels il s'agit avant tout pour les élèves de participer ${ }^{(11)}$, vont de pair avec une minoration des savoirs disciplinaires et de leurs contraintes épistémologiques et cognitives au profit des informations procédurales visant des compétences particulières.

Le discours d'accompagnement de l'effectuation des tâches collectives ou individuelles est produit dans cette même tonalité de connivence qui ne permet sans doute pas aux élèves de s'inscrire dans une production discursive et cognitive de savoir :

(11) Cet aspect du dialogue scolaire est largement développé par Bonnéry (2007). 
Ens: alors est-ce que vous êtes au point sur ce que c'est un segment?

Elève : une ligne qui est coupée par deux points

Ens : est-ce que ça vous va? demande l'enseignant sans attendre de réponse.

Nombre d'énoncés dans ces situations sont également elliptiques et dans ce même registre de connivence ; ils sont de ce fait non seulement peu compréhensibles pour de jeunes élèves, mais ne leur apportant pas suffisamment de ressources en langue grammatisée, en phrases introduisant aux formes écrites :

En Cours Préparatoire,

Ens : on va écrire avec un petit bout de Nabil (pour « avec une petite partie du texte proposé par Nabil »)

Ens : nous allons écrire une histoire qui ressemble à la chenille (pour « une histoire sur le modèle de l'histoire de la chenille qui fait des trous ").

L'absence d'un lexique de savoir est accrue lorsque, situation très fréquente en maternelle, des acquisitions nouvelles ou à consolider sont censées sous-tendre des activités présentées comme un jeu (justement avec le groupe des élèves faibles).

Cette description des caractéristiques dominantes du discours pédagogique actuel soulève la question générale des apprentissages auxquels il contribue. Que véhicule-t-il comme apprentissages et ceux-ci sont appropriés par quel type d'élèves? Elle permet de supposer qu'il ne peut avoir que peu d'impact sur la familiarisation des élèves à des usages langagiers qui leur permettent d'identifier une visée cognitive dans le travail scolaire et d'être en capacité de production d'un discours littéracié de recontextualisation. Ce discours et sa surcontextualisation qui entraîne la segmentation des savoirs et leur existence opportuniste ne les préparent pas davantage à être en capacité de mettre en relation des univers de savoir différents, des discours d'origine ou de statut différents. Pourtant, la régionalisation des savoirs actuels et les formes cognitives et langagières qui sont censées l'accompagner le supposent dès l'enseignement primaire. Nous en donnerons ici un rapide exemple tiré d'un manuel utilisé pour l'enseignement des sciences en $\mathrm{CM}^{(12)}$.

\subsection{Quand le travail quotidien de la classe suppose mises en relation et mobilisations de savoirs secondarisés, familiarité avec la lecture de documents écrits complexes}

Nous avions décrit au travers des modalités d'évaluation de la littéracie par PISA (Bautier et alii, 2006, Bautier \& Rayou, 2009) ce qui nous apparaissait déjà en 2000 comme une évolution curriculaire importante en ce qu'elle porte sur des raisonnements à partir du traitement de documents et correspondant à la mobilisation de connaissances largement non scolaires, voire expérientielles (à secondariser donc) et d'activités d'analyse et de commentaire. On retrouve depuis plusieurs années maintenant des traces de cette évolution dans les documents de travail pour les élèves du primaire. Nous en ferons ici une brève description qui vise à mettre au jour ce que les élèves doivent (en) comprendre, ce qu'ils doivent savoir (en) faire afin de pouvoir les utiliser avec les objectifs fixés. Compte tenu de l'exigence et du degré de complexité de tels documents, il est nécessaire que les élèves soient dans cette littéracie étendue sur laquelle nous avons insisté, ou puissent y être introduits par l'enseignant à cette occasion. Mais les interrogations sont gran-

(12) Les ateliers Hachette. Sciences expérimentales et technologie. 
des quant à cette possibilité après les observations de classes et les analyses du discours pédagogique.

Quelques caractéristiques de ce type d'ouvrage de sciences expérimentales et technologie mettent en évidence le curriculum latent à l'œuvre aujourd'hui.

- Dans le domaine des savoirs, les savoirs régionalisés, les « éducations à » (à l'environnement, à la santé, à l'informatique), conformément aux programmes, sont largement développés à partir des connaissances de la vie quotidienne plus ou moins médiatisée. Ils côtoient les savoirs disciplinaires classificatoires des sciences naturelles et expérimentales (la classification des espèces animales et végétales, par exemple). La distinction entre les deux domaines de savoir n'est pas apparente, l'entrée dans ces savoirs passant par une phase d'exploration qui repose pareillement sur des connaissances intuitives et des évidences culturelles.

- Les objectifs sont au demeurant explicitement des objectifs d'élaboration et de travail cognitif, puisqu'il s'agit de comprendre des notions, des phénomènes, des processus, des comportements, et d'aboutir à leur conceptualisation. Les documents sont là pour les susciter et supposent donc des activités de décontextualisation et de mise en relation d'éléments le plus souvent hétérogènes. Ici apparaît centrale la mobilisation d'un vocabulaire générique, fondé dans la pratique de l'écrit (au sens de littéracie) conceptualisant, qui permet justement de construire l'objet de savoir à partir de la pluralité des documents proposés et d'identifier le phénomène à traiter.

- Des documents très diversifiés (textes littéraires ou encyclopédiques, schémas, photographies, présentation d'expérience, dessins...) sont présentés sur une double page et accompagnés de questions diverses et d'encarts (coexistent un lexique, une rubrique "étonnant», un résumé synthèse à retenir, une consigne pour le « carnet de chercheur » et des questions de travail de haut niveau de complexité langagière et cognitive, alors même que les verbes des consignes sont justement, nous l'avons vu à propos du discours pédagogique, des verbes de la vie courante, des verbes qui ne « cadrent » pas, n'orientent pas l'activité cognitive attendue dans un champ disciplinaire particulier (certaines questions portent sur des faits stylistiques dans des textes de vulgarisation scientifique). L'usage du verbe « se passer » est ainsi très fréquent :

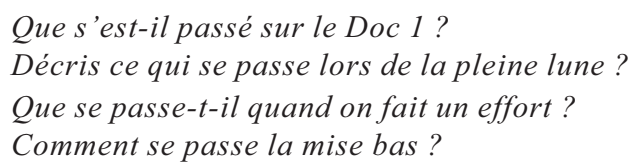

Autre exemple de question apparemment ouverte, mais en fait très précise dans une leçon titrée en français courant : comment les mammifères font-ils des petits? (la plupart des titres des leçons utilisent ce vocabulaire).

\section{D'où sortent les chatons?}

Ces questions, que le vocabulaire utilisé rend ouvertes, donnent lieu à des réponses de la part des élèves dans des domaines très divers et très différenciateurs du point de vue scolaire. Elles supposent pourtant une langue écrite précise et non quotidienne pour y répondre d'une façon qui identifie les phénomènes, ce qui est leur objectif :

Quelle forme présente la lune à chaque phase?

Décris les points communs et les différences entre les deux systèmes (une montgolfière du XVII ${ }^{\mathrm{e}}$ et une montgolfière moderne sont présentées par la reproduction d'une 
gravure et une photo, une légende mentionne le feu de paille pour l'une, le gaz pour l'autre).

Cet exemple, lorsqu'on lit les réponses que font les élèves, montre que la plupart n'ont d'autres solutions que de recopier la légende des reproductions, quand la question exige beaucoup plus, en particulier une écriture personnelle, comme c'est le cas dans PISA.

- Des activités cognitives doivent être mobilisées de façon prépondérante, celle de mise en relation ou d'inférence et celle de comparaison.

Il s'agit, par exemple, de comparer entre elles des bouteilles remplies d'eau froide recouvertes de différentes matières pour désigner laquelle (laine, poils, graisse...) isole le mieux, la modification de température de l'eau par rapport à la température initiale étant donnée par un thermomètre à côté de chaque schéma de bouteille.

Un tel dispositif expérimental suppose non seulement la comparaison entre les différentes bouteilles, ce que la plupart des élèves ne fait pas, considérant chaque bouteille pour elle-même, mais il suppose aussi que les élèves soient familiers du fonctionnement d'un thermomètre et de la nature des informations qu'il donne. Tel n'est pas le cas dans les classes de ZEP étudiées ${ }^{(13)}$. On rencontre ici les effets de la connivence culturelle supposée : en effet, sauf exception, tous les élèves ont vu dans leur vie un thermomètre et savent qu'il sert à connaître la température extérieure pour décider comment on s'habille (sic); mais cette connaissance par expérience n'est ici d'aucune utilité. Il faut en effet connaître d'autres usages et la leçon ne porte pas sur, ni ne rappelle, l'usage et le fonctionnement de cet objet familier.

- Lire l'ensemble de la double page, ou tout au moins en prendre connaissance rapidement, permet d'être aidé dans la compréhension de chaque étape, alors même que les élèves, les plus faibles en particulier, et souvent en y étant engagés par le maître, font une lecture et les activités au pas à pas.

$C$ 'est donc une connivence culturelle qui est largement à l'œuvre dans les documents proposés.

Il en est ainsi quand les photos présentent un astronome amateur observant le ciel la nuit avec un télescope et à côté un touareg seul marchant dans le désert en plein jour (la question porte sur les possibilités de l'homme pour se repérer dans le temps et l'espace). Certains élèves identifient le télescope et son usage quand d'autres n'en connaissent même pas l'existence... Comme pour la référence au fonctionnement du thermomètre, l'implicite va porter ici sur le fonctionnement du télescope ; même s'il est indiqué par l'enseignant en une phrase (ça sert à regarder les étoiles et les planètes), sa méconnaissance ne permet pas aux élèves de se servir de cet indice. Il en est de même de la référence à l'ombre portée par le touareg sur le sable.

Les inégalités entre élèves sont très grandes dans de telles situation de travail, alors même que la forme du discours pédagogique dominante ne permet pas de les combler, ne permet pas d'introduire les élèves à l'ensemble des activités cognitives, des modalités de description et d'explicitation des phénomènes qu'ils sont pourtant censés mettre en œuvre.

(13) Thèse de doctorat de P. Savina en préparation, dir. E. Bautier, université de Paris 8. 


\section{Conclusion}

La description, que nous avons présentée, d'un discours pédagogique actuellement dominant nous semble éloigner les élèves d'une socialisation langagière et cognitive qui les inscrirait dans la familiarité avec la littéracie étendue supposée par le fonctionnement de l'école aujourd'hui. Les difficultés qui s'ensuivent pour certains élèves au moins vont dans le sens d'un accroissement des inégalités, accroissement renforcé par la mise en œuvre différenciée de ce discours pédagogique selon le niveau réel ou représenté des élèves. En effet, alors qu'avec les classes jugées bonnes, la langue standard est souvent présente et produite par l'enseignant, plus les élèves sont considérés comme ayant des difficultés scolaires et/ou étant de milieux populaires, plus les enseignants inscrivent leur intervention et le dialogue sur le registre quotidien et horizontal ${ }^{(14)}$, plus les phrases sont inachevées et peu formelles.

L'évolution curriculaire et langagière que nous venons de décrire interroge les possibilités pour les élèves à produire un texte de savoir. Tout semble se passer comme si actuellement, les élèves étaient davantage entraînés à répondre à des questions, dire un sentiment ou une opinion en écho à tel ou tel texte, qu'habitués à produire un texte faisant dialoguer des savoirs au sein d'une discipline identifiée à l'aide d'une familiarité avec une langue et ses usages littéraciés.

\section{Bibliographie}

Amigues, R. \& Zerbato-Poudou, M.-T. (2001) : Comment l'enfant devient élève. Les apprentissages à l'école maternelle, Paris, Retz.

Astolfi, J.-P. PETERfalvi, G. et VÉRIN, A. (1998), Comment les enfants apprennent les sciences, , Paris, Retz.

BAKHTINE, M. (trad. franç. 1984) : : Esthétique de la création verbale, Paris, Gallimard, 1984, pp. 265-272.

B AUTIER, É. dir. (2006) : Apprendre l'école et apprendre à l'école. Des risques de construction des inégalités dès la maternelle, contributeurs : É. Bautier, O. Burger, C. Catteau, S. Chevillard, C. Joigneaux, J. Le Breton, C. Passerieux, C. Thouny, Lyon, Chronique sociale.

BAUTIER, É. (2005) : « Mobilisation de soi, exigences langagières scolaires et processus de différenciation », Langage et société, n¹11, 2005, pp. 51-72.

- (2005): «Formes et activités scolaires, secondarisation, reconfiguration, différenciation sociale », in N. Ramognino, P. Vergès, (eds), Le Français hier et aujourd'hui. Politiques de la languie et apprentissages scolaires. Études offertes à V. Isambert-Jamati, Publications de l'Université de Provence.

(14) Il ne s'agit pas ici, on l'aura compris, des usages par les enseignants d'une langue «jeune » qui conduit à qualifier les exercices et travaux à effectuer de «cool» ou de «sympa ». 
BAUtier, É. GoigouX, R. (2004) : « Difficultés d'apprentissage, processus de secondarisation et pratiques enseignantes : une hypothèse relationnelle», Revue Française de Pédagogie, ${ }^{\circ} 148$, pp. 89-100.

B AUtIER, É. \& RAYOU, P. (2009) : Les inégalités d'apprentissage. Programmes, pratiques et malentendus scolaires, Paris, P.U.F.

BAUTIER, É. \& ROCHEX, J.-Y. (2004) : «Activité conjointe ne signifie pas significations partagées », in C. Moro et R. Rickenmann (eds), Situation éducative et significations, Raisons Éducatives, Bruxelles, De Boeck.

BAUtier, É., CRINON, J., RAYOU, P., ROChEX, J.-Y. (2006) : «Performances en littéracie, modes de faire et univers mobilisés par les élèves : analyses secondaires de l'enquête PISA 2000 », Revue Française de pédagogie, n¹57, pp. 85-101.

Bernstein, B. (2007) : Pédagogie, contrôle symbolique et identité, Laval, P.U.Laval.

BONNÉRY, S. (2007) : Comprendre l'échec scolaire. Élèves en difficulté et dispositifs pédagogiques, Paris, La Dispute.

CAFFIEUX, C. (2007) : L'entrée dans l'écrit : l'influence des pratiques d'enseignement à l'école maternelle, ULB, Bruxelles, sous la direction de $\mathrm{B}$. Rey.

FORQUIN, J.-C. (2008) : Sociologie du curriculum, Rennes, P.U.R.

JoIGNEAUX, C. (2009) : Des processus de différenciation dès l'école maternelle. Historicités plurielles et inégalité scolaire. Thèse de doctorat, juillet 2009, université de Paris 8, sous la direction de J.-Y. Rochex.

KARA, M. \& Privat, J.-M. (2006) : Co-direction du n 131-132 de Pratiques, « La littératie. Autour de Jack Goody », 256 pages, Cresef, Metz.

LAHIRE, B. (2008) : La raison scolaire, Rennes, P.U.R.

MATON, K. (2008) : "Gravité sémantique et apprentissage segmenté », in D. Frandji \& P. Vitale, dirs, Actualité de Basil Bernstein, Rennes, P.U.R.

Reuter, Y. (2006) : « À propos des usages de Goody en didactique », Pratiques, n`131132.

Young, M.F.D. (1998) : The Curriculum of the future. From the NSE to a critical theory of Learning, London, Falmer Press.

— (2001): «Du "curriculum en tant que construction sociale" à la "spécialisation intégrative". Quelques réflexions sur la sociologie du curriculum », Revue française de pédagogie, ${ }^{\circ} 135$, pp. 29-34.

- (2008) : Bringing knowledge back in, London, New York, Routledge. 